\title{
Exciton spectrum in multi-shell hexagonal semiconductor nanotube
}

\author{
O.M. Makhanets*, V.I. Gutsul, N.R. Tsiupak, O.M. Voitsekhivska \\ Chernivtsi National University, 2 Kotsyubinsky Str., 58012 Chernivtsi, Ukraine \\ Received March 16, 2012, in final form May 29, 2012
}

The theory of exciton spectrum in multi-shell hexagonal semiconductor nanotube is developed within the effective masses and rectangular potentials approximations using the method of effective potential. It is shown that the exciton binding energy for all states non-monotonously depends on the inner wire diameter, approaching several minimal and maximal magnitudes. The obtained theoretical results explain well the experimental positions of luminescence peaks for $\mathrm{GaAs} / \mathrm{Al}_{0.4} \mathrm{Ga}_{0.6}$ As nanotubes.

Key words: hexagonal nanotube, quantum wire, exciton spectrum

PACS: 73.21.Hb, 78.67.Ch, 78.67.Lt

\section{Introduction}

The semiconductor quantum wires are theoretically and experimentally studied during more than twenty years. The improved methods of their growth (molecular beam, gas phase and metal organic epitaxy) gave an opportunity to produce arrays of quantum nanowires with a radial heterostructure [1, 2].

On the one hand, the heterostructure perpendicular to the quantum wire axis can localize the charge carriers inside the inner wire, thus decreasing the surface scattering [3]. On the other hand, this allows a guided change of spectral parameters of quasi-particles (electrons, excitons, phonons) depending on nanostructure geometric parameters. The unique properties of quasi-particles make it possible to utilize such systems as the basic elements of tunnel nanodiodes, nanotransistors with high mobility of electrons, effective light emitting devices, photo electric transformers, nanosensors used for the diagnostics of biological and chemical compositions [4].

One of the variety of quantum wires with radial heterostructure is a semiconductor nanotube intensively investigated recently. The single (with one quantum well for quasi-particles) [5, 6] and complex multi-shell (with several wells) [7, 8] hexagonal nanotubes are already produced experimentally using different semiconductor materials.

The investigation of exciton binding energy for these structures encounters serious mathematical problems connected with the necessary correlation of spherical symmetry of Coulomb potential describing the electron-hole interaction and non-spherical symmetry of a system itself. Therefore, the exciton spectrum is often studied within different and rather simple variational methods [9, 10], which are capable of quite well describing only the exciton ground state. The method of effective potentials turns out to be more informative. The theory of exciton spectrum in single cylindrical semiconductor quantum wires has already been developed [11, 12] using this method.

In this paper we propose one of the theoretical approaches to the solution of the problem of exciton spectrum in multi-shell hexagonal semiconductor nanotube. The theory is developed within the model of effective masses and rectangular potentials using the method of effective potentials. We study the parameters of exciton spectra depending on the geometrical parameters of a nanostructure and compare the numerical results obtained for the exciton energies with the positions of luminescence peaks observed experimentally [8].

\footnotetext{
*E-mail: ktf@chnu.edu.ua
} 


\section{Theory of exciton spectra in multi-shell hexagonal semiconductor na- notube}

The experimentally grown nanostructure [8] - the multi-shell hexagonal nanotube is theoretically studied. It consists of hexagonal semiconductor quantum wire ("0"), thin barrier-shell ("1") and nanotube (“2”) embedded into the outer medium (“3”). The transversal cross-section of nanostructure is shown in figure 1.

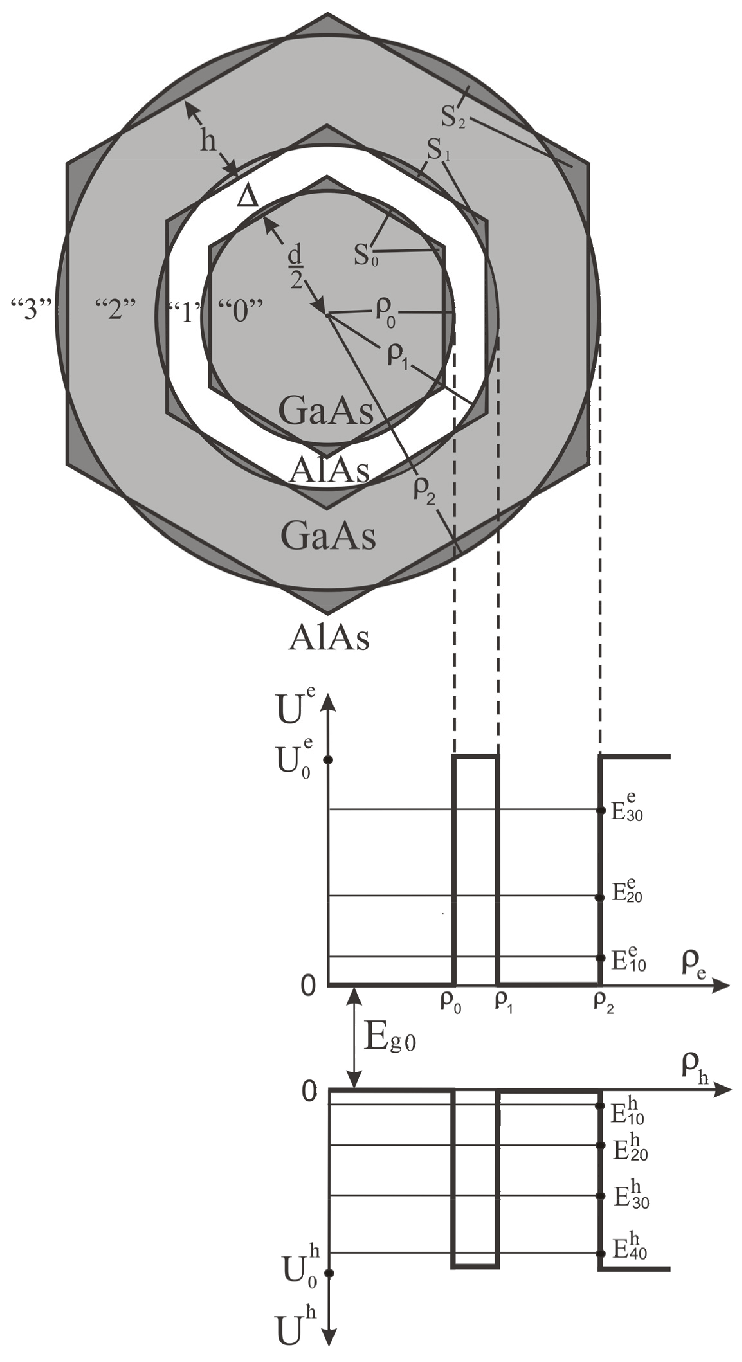

Figure 1. Transversal cross-section of nanostructure and its energy scheme.

Within the assumption that the lattice and dielectric constants of nanostructure compositions do not differ much, we use the effective masses $\left(m^{\mathrm{e}, \mathrm{h}}\right)$ and rectangular potentials $\left(U^{\mathrm{e}, \mathrm{h}}\right)$ models to calculate the electron, hole and exciton spectra.

We solve the Schrödinger equation for the exciton

$$
\hat{H}_{\mathrm{ex}}\left(\vec{r}_{\mathrm{e}}, \vec{r}_{\mathrm{h}}\right) \Psi_{\mathrm{ex}}\left(\vec{r}_{\mathrm{e}}, \vec{r}_{\mathrm{h}}\right)=E_{\mathrm{ex}} \Psi_{\mathrm{ex}}\left(\vec{r}_{\mathrm{e}}, \vec{r}_{\mathrm{h}}\right)
$$

with the Hamiltonian

$\hat{H}_{\mathrm{ex}}\left(\vec{r}_{\mathrm{e}}, \vec{r}_{\mathrm{h}}\right)=E_{g 0}+\hat{H}^{\mathrm{e}}\left(\vec{r}_{\mathrm{e}}\right)+\hat{H}^{\mathrm{h}}\left(\vec{r}_{\mathrm{h}}\right)+V\left(\left|\vec{r}_{\mathrm{e}}-\vec{r}_{\mathrm{h}}\right|\right)$,

where $E_{g_{0}}$ is the band gap of the nanotube,

$$
\begin{aligned}
& \hat{H}^{(p)}=-\frac{\hbar^{2}}{2}\left[\vec{\nabla}_{\rho_{p}, \varphi_{p}} \frac{1}{m^{p}\left(\rho_{p}, \varphi_{p}\right)} \vec{\nabla}_{\rho_{p}, \varphi_{p}}\right. \\
& \left.+\frac{1}{m^{p}\left(\rho_{p}, \varphi_{p}\right)} \frac{\partial^{2}}{\partial z_{p}^{2}}\right]+U^{p}\left(\rho_{p}, \varphi_{p}\right), \quad(p=\mathrm{e}, \mathrm{h})
\end{aligned}
$$

are the Hamiltonians of non-interacting electron and hole in cylindrical coordinates $(\rho, \varphi, z)$,

$$
V\left(\left|\vec{r}_{\mathrm{e}}-\vec{r}_{\mathrm{h}}\right|\right)=-\frac{e^{2}}{\varepsilon\left(\vec{r}_{\mathrm{e}}, \vec{r}_{\mathrm{h}}\right)\left|\vec{r}_{\mathrm{e}}-\vec{r}_{\mathrm{h}}\right|}
$$

is the potential energy of their interaction.

The equation (1) with Hamiltonian (2) cannot be solved exactly due to the complicated dependence of the potential energy (4) and physical characteristics $\left(m^{p}, U^{p}\right)$ of the quasi-particles on geometrical parameters of hexagonal nanotube.

An approximated solution of the problem is performed within two stages [13, 14]. Considering that the energy of electron-hole interaction is much smaller than the energy of their size quantization, we first obtain the latter for the model of hexagonal nanotube and then take into account the potential of interaction of both quasi-particles (4).

Thus, the stationary Schrödinger equations for the non-interacting electron and hole are solved in a cylindrical coordinate system

$$
\hat{H}^{(p)}\left(\vec{r}_{p}\right) \psi^{(p)}\left(\vec{r}_{p}\right)=E^{(p)} \psi^{(p)}\left(\vec{r}_{p}\right), \quad(p=\mathrm{e}, \mathrm{h})
$$

It is clear that the effective masses $\left[m^{(\mathrm{e}, \mathrm{h})}\right]$ and potential energies $\left[U^{(\mathrm{e}, \mathrm{h})}\right]$ as functions of $\rho, \varphi$ variables have a hexagonal symmetry in the plane perpendicular to the nanotube axis. Thus, the variables are not separated and the equations (5) cannot be solved exactly. The approximated solution is found within the 
Bethe variational method. In the Hamiltonian (3) the main term is introduced. The magnitudes of $m^{(\mathrm{e}, \mathrm{h})}$ and $U^{(\mathrm{e}, \mathrm{h})}$ in this term are the functions of a radial variable $\rho$. I.e., hexagons are replaced by circles of respective radii: $\rho_{0}, \rho_{1}=\rho_{0}+\Delta, \rho_{2}=\rho_{0}+\Delta+h$. Within this approach, the effective masses, potential energies of an electron and a hole as well as dielectric constants depend on the variable in the following way

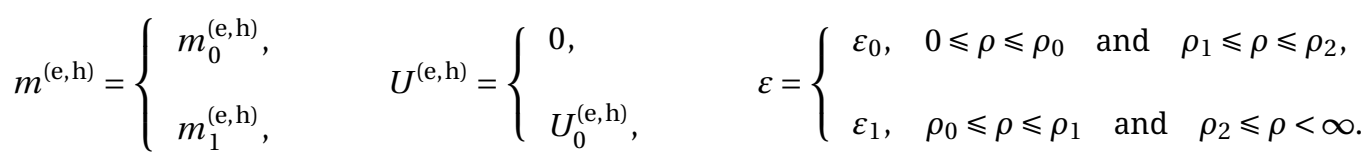

The differences between the respective masses $m^{(\mathrm{e}, \mathrm{h})}(\rho, \varphi)$ and $m^{(\mathrm{e}, \mathrm{h})}(\rho)$, and potentials $U^{(\mathrm{e}, \mathrm{h})}(\rho, \varphi)$ and $U^{(\mathrm{e}, \mathrm{h})}(\rho)$, arising as a result of approximation, are taken into account in the Hamiltonian as a perturbation. Herein, the radius of the smallest circle $\rho_{0}$ is considered as a variational parameter according to Bethe method.

Now, the Hamiltonian $\left[\hat{H}^{(\mathrm{e}, \mathrm{h})}\right]$ of the uncoupling electron and hole is written as follows:

$$
\hat{H}^{(p)}=\hat{H}_{0}^{(p)}+\Delta \hat{H}^{(p)}, \quad(p=\mathrm{e}, \mathrm{h}),
$$

where

$$
\hat{H}_{0}^{(p)}=-\frac{\hbar^{2}}{2}\left[\vec{\nabla}_{\rho, \varphi}^{(p)} \frac{1}{m^{(p)}(\rho)} \vec{\nabla}_{\rho, \varphi}^{(p)}+\frac{1}{m^{(p)}(\rho)} \frac{\partial^{2}}{\partial z^{2}}\right]+U^{(p)}(\rho)
$$

is the main part of the Hamiltonian which describes the electron and hole with effective masses $m^{(\mathrm{e}, \mathrm{h})}(\rho)$ and potential energies $U^{(\mathrm{e}, \mathrm{h})}(\rho)$ in a multi-shell cylindrical nanostructure.

The correction that takes into account the difference between the exact $\left[\hat{H}^{(p)}\right]$ and approximated $\left[\hat{H}_{0}^{(p)}\right]$ Hamiltonians

$$
\begin{aligned}
\Delta \hat{H}^{(p)=} & U^{(p)}(\rho, \varphi)-U^{(p)}(\rho) \\
& +\frac{\hbar^{2}}{2}\left\{\vec{\nabla}_{\rho, \varphi}\left[\frac{1}{\mu^{(p)}(\rho)}-\frac{1}{\mu^{(p)}(\rho, \varphi)}\right] \vec{\nabla}_{\rho, \varphi}-\left[\frac{1}{\mu^{(p)}(\rho)}-\frac{1}{\mu^{(p)}(\rho, \varphi)}\right] \frac{\partial^{2}}{\partial z^{2}}\right\}
\end{aligned}
$$

is further considered as a perturbation.

The Schrödinger equation with Hamiltonian (8) is solved exactly. Herein, we obtain analytical expressions for wave functions $\left[\psi_{n_{\rho} m}^{(p)}\left(\rho_{0}, \vec{r}_{p}\right)=\varphi_{n_{\rho} m}^{(p)}\left(\rho_{0}, \vec{\rho}_{p}\right) \exp \left(\mathrm{i} k_{p} z_{p}\right)\right]$ and electron (hole) energy spectrum $\left[E_{n_{\rho} m}^{(0)(p)}\left(\rho_{0}, k\right)\right]$ as a function of $\rho_{0}$ variational parameter in zero approximation of a perturbation method.

Further, according to Bethe method, we calculate the corrections of the first order to the energies of the both quasi-particles (e, h) as functions of $\rho_{0}$

$$
\delta E_{n_{\rho} m}^{(p)}\left(\rho_{0}, k\right)=6 \int_{-L / 2}^{L / 2} \sum_{j=0}^{2} \int_{S_{j}} \Psi_{n_{\rho} m}^{(p) *}\left(\rho_{0}, \vec{r}_{p}\right) \Delta \hat{H} \Psi_{n_{\rho} m}^{(p)}\left(\rho_{0}, \vec{r}_{p}\right) \mathrm{d}^{3} \vec{r}_{p}, \quad(p=\mathrm{e}, \mathrm{h}),
$$

where $L$ denotes the effective region of a quasi-particle movement along the axial axis of a nanotube.

We should note that due to the evident analytical properties of a perturbation Hamiltonian (9), integration over $\rho, \varphi$ variables in the expression (10) is performed only over $S_{j}$ regions located between the respective hexagons and approximating circles (shadowed regions in figure 1).

Now, without taking the electron-hole interaction into account, exciton energy spectrum is obtained from the condition of functional minimum

$$
\begin{aligned}
\varepsilon_{n_{\rho}^{\mathrm{h}} m^{\mathrm{h}}}^{n_{\rho}^{\mathrm{e}} m^{\mathrm{e}}} & =E_{g_{0}}+E_{n_{\rho}^{\mathrm{e}} m^{\mathrm{e}}}+E_{n_{\rho}^{\mathrm{h}} m^{\mathrm{h}}} \\
& =E_{g_{0}}+\min \left[E_{n_{\rho}^{\mathrm{e}} m^{\mathrm{e}}}^{(0)}\left(\rho_{0}\right)+\delta E_{n_{\rho}^{\mathrm{e}} m^{\mathrm{e}}}\left(\rho_{0}\right)+E_{n_{\rho}^{\mathrm{h}} m^{\mathrm{h}}}^{(0)}\left(\rho_{0}\right)+\delta E_{n_{\rho}^{\mathrm{h}} m^{\mathrm{h}}}\left(\rho_{0}\right)\right]_{\rho_{0}=\bar{\rho}_{0}},
\end{aligned}
$$

realized at $\rho_{0}=\overline{\rho_{0}}$. 
The electron and hole binding energies $\left(\Delta E_{n_{\rho}^{\mathrm{h}} m^{\mathrm{h}}}^{n_{\mathrm{e}}^{\mathrm{e}} m^{\mathrm{e}}}\right)$ in the respective states are calculated in the following way. In the space of quantum wire (“0”) or nanotube (“2”) there is performed an averaging of the potential interacting energy (4) at the electron and hole wave functions describing their movement in the plane perpendicular to the axial axis in the system of mass center $\left[z=z_{\mathrm{e}}-z_{\mathrm{h}}, Z=\left(z_{\mathrm{e}} m_{0}^{\mathrm{e}}+\right.\right.$ $\left.\left.z_{\mathrm{h}} m_{0}^{\mathrm{h}}\right) /\left(m_{0}^{\mathrm{e}}+m_{0}^{\mathrm{h}}\right)\right]$

$$
V_{n_{\rho}^{\mathrm{h}} m^{\mathrm{h}}}^{n_{\rho}^{\mathrm{e}} m^{\mathrm{e}}}(z)=\frac{e^{2}}{\varepsilon_{0}} \int \mathrm{d} \vec{\rho}_{\mathrm{e}} \mathrm{d} \vec{\rho}_{\mathrm{h}} \frac{\left|\varphi_{n_{\rho}^{\mathrm{e}} m^{\mathrm{e}}}\left(\bar{\rho}_{0}, \vec{\rho}_{\mathrm{e}}\right) \varphi_{n_{\rho}^{\mathrm{h}} m^{\mathrm{h}}}\left(\bar{\rho}_{0}, \vec{\rho}_{\mathrm{h}}\right)\right|^{2}}{\sqrt{\left(\vec{\rho}_{\mathrm{e}}-\vec{\rho}_{\mathrm{h}}\right)^{2}+z^{2}}}
$$

We should note that this potential describes not only the Coulomb interaction between the electron and hole along the $\mathrm{Oz}$ axis but also "effectively" takes it into account at the transversal plane.

Now, the Hamiltonian (2) takes the form

$$
\hat{H}_{\mathrm{ex}}=-\frac{\hbar^{2}}{2 M} \frac{\partial^{2}}{\partial Z^{2}}-\frac{\hbar^{2}}{2 \mu} \frac{\partial^{2}}{\partial z^{2}}-V_{n_{\rho}^{\mathrm{h}} m^{\mathrm{h}}}^{n_{\mathrm{e}}^{\mathrm{e}} m^{\mathrm{e}}}(z)+\varepsilon_{n_{\rho}^{\mathrm{h}} m^{\mathrm{h}}}^{n_{\mathrm{e}}^{\mathrm{e}} m^{\mathrm{e}}} .
$$

Here,

$$
M=m_{0}^{\mathrm{e}}+m_{0}^{\mathrm{h}}, \quad \mu=\frac{m_{0}^{\mathrm{e}} m_{0}^{\mathrm{h}}}{m_{0}^{\mathrm{e}}+m_{0}^{\mathrm{h}}}
$$

are the effective mass of an exciton generally moving in the longitudinal direction and its reduced mass, respectively.

From the expression (13) it is clear that the movement of the mass centre of an exciton along the $O Z$ axis is separated in such a way that the energy $E_{P}$ and wave function $\left[\Psi_{P}(Z)\right]$ of an exciton longitudinal movement are as follows:

$$
E_{P}=\frac{P^{2}}{2 M}, \quad \Psi_{P}(Z)=\frac{1}{\sqrt{2 \pi \hbar}} \exp \left(\frac{\mathrm{i} P Z}{\hbar}\right) .
$$

The Schrödinger equation separately for $z$-th component cannot be solved exactly. In order to obtain its approximated solution, in the Hamiltonian (13) we add and subtract the potential

$$
V(z)=-\frac{e^{2}}{\varepsilon_{0}} \frac{1}{(\beta+|z|)}
$$

with variational parameter $\beta$.

Such a potential, on the one hand, has the main properties of a potential of electron-hole interaction (12) and, thus, together with the kinetic energy of $z$-th component, provides the energy of the bound state $E_{n_{z}}$ and, on the other hand, contrary to the potential (12) provides a rather small magnitude within the conception of perturbation theory

$$
\Delta V_{n_{\rho}^{\mathrm{h}} m^{\mathrm{h}}}^{n_{\rho}^{\mathrm{e}} m^{\mathrm{e}}}=\frac{e^{2}}{\varepsilon_{0}}\left[\frac{1}{\beta+|z|}-V_{n_{\rho}^{\mathrm{h}} m^{\mathrm{h}}}^{n_{\rho}^{\mathrm{e}} m^{\mathrm{e}}}(z)\right] .
$$

The Schrödinger equation

$$
\hat{H}_{z} \Psi_{n_{z}}(z)=E_{n_{z}} \Psi_{n_{z}}(z)
$$

with the Hamiltonian

$$
\hat{H}_{z}=-\frac{\hbar^{2}}{2 \mu} \frac{\partial^{2}}{\partial z^{2}}-\frac{e^{2}}{\varepsilon_{0}} \frac{1}{(\beta+|z|)}
$$

is solved exactly [15] and the wave function is obtained as

$$
\Psi_{n_{z}}(z)=A \exp [-\chi(z+\beta)] F\left[-\frac{v}{2 \chi} ; 0 ; 2 \chi(z+\beta)\right] .
$$


Here,

$$
v=\frac{2 \mu}{\hbar^{2}} \frac{e^{2}}{\varepsilon_{0}}, \quad \chi^{2}=\frac{2 \mu}{\hbar^{2}} E_{n_{z}},
$$

$A$ is the normality constant and $F$ is the confluent hyper-geometrical function.

The equation (18) with the Hamiltonian (19) is symmetrical with respect to the replacement $z \rightarrow-z$. Thus, its solutions should be even or odd. This brings about two boundary conditions

$$
\begin{cases}\left.\frac{\partial \Psi_{n_{z}}(z)}{\partial z}\right|_{z=0}=0, & \Psi_{n_{z}}-\text { even; } \\ \Psi_{n_{z}}(0)=0, & \Psi_{n_{z}} \text { - odd }\end{cases}
$$

consistently defining the energy spectrum $E_{n_{z}}$.

Now, the exciton energy, as a function of variational parameter $\beta$, is presented by the expression

$$
E_{n_{\rho}^{\mathrm{h}} m^{\mathrm{h}}}^{n_{\mathrm{\textrm {h }}}^{\mathrm{e}} m^{\mathrm{e}}}\left(n_{z}, P, \beta\right)=E_{n_{\rho}^{\mathrm{e}} m^{\mathrm{e}}}+E_{n_{\rho}^{\mathrm{h}} m^{\mathrm{h}}}+\frac{P^{2}}{2 M}+E_{g 0}+\Delta E_{n_{\rho}^{\mathrm{h}} m^{\mathrm{h}}}^{n_{\rho}^{\mathrm{e}} m^{\mathrm{e}}}\left(n_{z}, \beta\right)
$$

and the wave functions of zero approximation

$$
\Psi_{n_{\rho}^{\mathrm{h}} m^{\mathrm{h}}}^{n_{\rho}^{\mathrm{e}} m^{\mathrm{e}}}\left(n_{z}, P, \beta\right)=\Psi_{P}(Z) \Psi_{n_{z}}(\beta, z) \varphi_{n_{\rho}^{\mathrm{e}} m^{\mathrm{e}}}\left(\overline{\rho_{0}}, \rho_{\mathrm{e}}, \varphi_{\mathrm{e}}\right) \varphi_{n_{\rho}^{\mathrm{h}} m^{\mathrm{h}}}\left(\overline{\rho_{0}}, \rho_{\mathrm{h}}, \varphi_{\mathrm{h}}\right)=\left|\begin{array}{c}
n_{\rho}^{\mathrm{e}} m^{\mathrm{e}} \\
n_{\rho}^{\mathrm{h}} m^{\mathrm{h}}
\end{array} n_{z} P\right\rangle .
$$

The binding energy [ $\left.\Delta E_{n_{\rho}^{\mathrm{h}} m^{\mathrm{h}}}^{n_{\mathrm{\textrm {e }}}^{\mathrm{e}} m^{\mathrm{e}}}\left(n_{z}, \beta\right)\right]$ of an exciton in the expression (23), naturally, consists of the energy of the bound state $E_{n_{z}}$ along $O z$ axis and the correction $\left[\delta E_{n_{\rho}^{\mathrm{h}} m^{\mathrm{h}}}^{n_{\mathrm{h}}^{\mathrm{e}} m^{\mathrm{e}}}\left(n_{z}, \beta\right)\right]$ calculated as a diagonal matrix element of a perturbation operator (17) at the wave functions (24)

$$
\begin{aligned}
\Delta E_{n_{\rho}^{\mathrm{h}} m^{\mathrm{h}}}^{n_{\rho}^{\mathrm{e}} m^{\mathrm{e}}}\left(n_{z}, \beta\right) & =E_{n_{z}}(\beta)+\delta E_{n_{\rho}^{\mathrm{h}} m^{\mathrm{h}}}^{n_{\rho}^{\mathrm{e}} m^{\mathrm{e}}}\left(n_{z}, \beta\right), \\
\delta E_{n_{\rho}^{\mathrm{h}} m^{\mathrm{h}}}^{n^{\mathrm{e}} m^{\mathrm{e}}} & \left(n_{z}, \beta\right)=\frac{e^{2}}{\varepsilon_{0}}\left\langle n_{z}\left|\left(\frac{1}{\beta+|z|}-V_{n_{\rho}^{\mathrm{h}} m^{\mathrm{h}}}^{n^{\mathrm{e}} m^{\mathrm{e}}}(z)\right)\right| n_{z}\right\rangle .
\end{aligned}
$$

Having the magnitude $\bar{\beta}$ ensuring the minimum of $\Delta E_{n_{\rho}^{\mathrm{h}} m^{\mathrm{h}}}^{n_{\rho}^{\mathrm{e}} m^{\mathrm{e}}}\left(n_{z}, \bar{\beta}\right)=\Delta E_{n_{\rho}^{\mathrm{h}} m^{\mathrm{h}}}^{n^{\mathrm{e}} m^{\mathrm{e}}}\left(n_{z}\right)$, we obtain final expressions for the energies

$$
E_{n_{\rho}^{\mathrm{h}} m^{\mathrm{h}}}^{n_{\mathrm{\textrm {e }}}^{\mathrm{e}} m^{\mathrm{e}}}\left(n_{z}, P\right)=E_{n_{\rho}^{\mathrm{e}} m^{\mathrm{e}}}+E_{n_{\rho}^{\mathrm{h}} m^{\mathrm{h}}}+\frac{P^{2}}{2 M}+E_{g 0}+\Delta E_{n_{\rho}^{\mathrm{h}} m^{\mathrm{h}}}^{n_{\mathrm{h}}^{\mathrm{e}} m^{\mathrm{e}}}\left(n_{z}\right)
$$

and wave functions

$$
\Psi_{n_{\rho}^{\mathrm{h}} m^{\mathrm{h}}}^{n_{\mathrm{e}}^{\mathrm{e}} m^{\mathrm{e}}}\left(n_{z}, P\right)=\Psi_{P}(Z) \Psi_{n_{z}}(z) \varphi_{n_{\rho}^{\mathrm{e}} m^{\mathrm{e}}}\left(\overline{\rho_{0}}, \rho_{\mathrm{e}}, \varphi_{\mathrm{e}}\right) \varphi_{n_{\rho}^{\mathrm{h}} m^{\mathrm{h}}}\left(\overline{\rho_{0}}, \rho_{\mathrm{h}}, \varphi_{\mathrm{h}}\right)
$$

of the exciton in a multi-shell hexagonal nanotube.

The electron and hole wave functions are used for the evaluation of the intensities of the interband optical quantum transitions according to the formula [16]

$$
I_{n_{\rho}^{\mathrm{h}} m^{\mathrm{h}}}^{n_{\rho}^{\mathrm{e}} m^{\mathrm{e}}} \sim\left|\iint \varphi_{n_{\rho}^{\mathrm{e}} m^{\mathrm{e}}}^{*}\left(\overline{\rho_{0}}, \vec{\rho}\right) \varphi_{n_{\rho}^{\mathrm{h}} m^{\mathrm{h}}}\left(\overline{\rho_{0}}, \vec{\rho}\right) \mathrm{d} \vec{\rho}\right|^{2}
$$

A further calculation of the spectrum and the analysis of its properties was performed using numeric methods for the $\mathrm{GaAs} / \mathrm{Al}_{0.4} \mathrm{Ga}_{0.6}$ As multi-shell hexagonal nanotube grown experimentally [8]. 


\section{Discussion of results}

Exciton spectrum as a function of a nanostructure geometrical parameters is studied for $\mathrm{GaAs} / \mathrm{Al}_{0.4} \mathrm{Ga}_{0.6} \mathrm{As}$ multi-shell hexagonal nanotube having physical parameters [7, 8, 17]: $m_{0}^{\mathrm{e}}=0.063 m_{0}$, $m_{1}^{\mathrm{e}}=0.096 m_{0}, m_{0}^{\mathrm{h}}=0.51 m_{0}, m_{1}^{\mathrm{h}}=0.61 m_{0}, U_{0}^{\mathrm{e}}=297 \mathrm{meV}, U_{0}^{\mathrm{h}}=224 \mathrm{meV}, E_{g 0}=1520 \mathrm{meV}, \varepsilon_{0}=10.89$, ( $m_{0}$ is the pure electron mass in vacuum); $a_{\mathrm{GaAs}}=5.65 \AA$ is the lattice constant of GaAs.

In figure 2 the electron energy $E_{n_{\rho} 0}^{\mathrm{e}}(\mathrm{a})$, heavy hole energy $E_{n_{\rho} 0}^{\mathrm{h}}(\mathrm{b})$, exciton binding energy $\Delta E_{n_{\rho} 0}^{n_{\rho}^{\mathrm{e}} 0}$ (c) and exciton energy $E_{n_{\rho}^{\mathrm{h}} 0}^{n_{\rho}^{\mathrm{e}} 0}(\mathrm{~d})$ are presented as functions of the inner wire GaAs diameter $d$ at $P=0, n_{z}=1$, and experimental magnitudes for the barrier-shell size: $\Delta=4 \mathrm{~nm}$ and nanotube width: $h=4 \mathrm{~nm}$ [8]. In figures 2 (a), (b) one can see only two energy levels at $d=0$. These levels coincide with the ones obtained for a hexagonal nanotube (GaAs) embedded into $\mathrm{Al}_{0.4} \mathrm{Ga}_{0.6} \mathrm{As}$, which is proven by physical considerations.
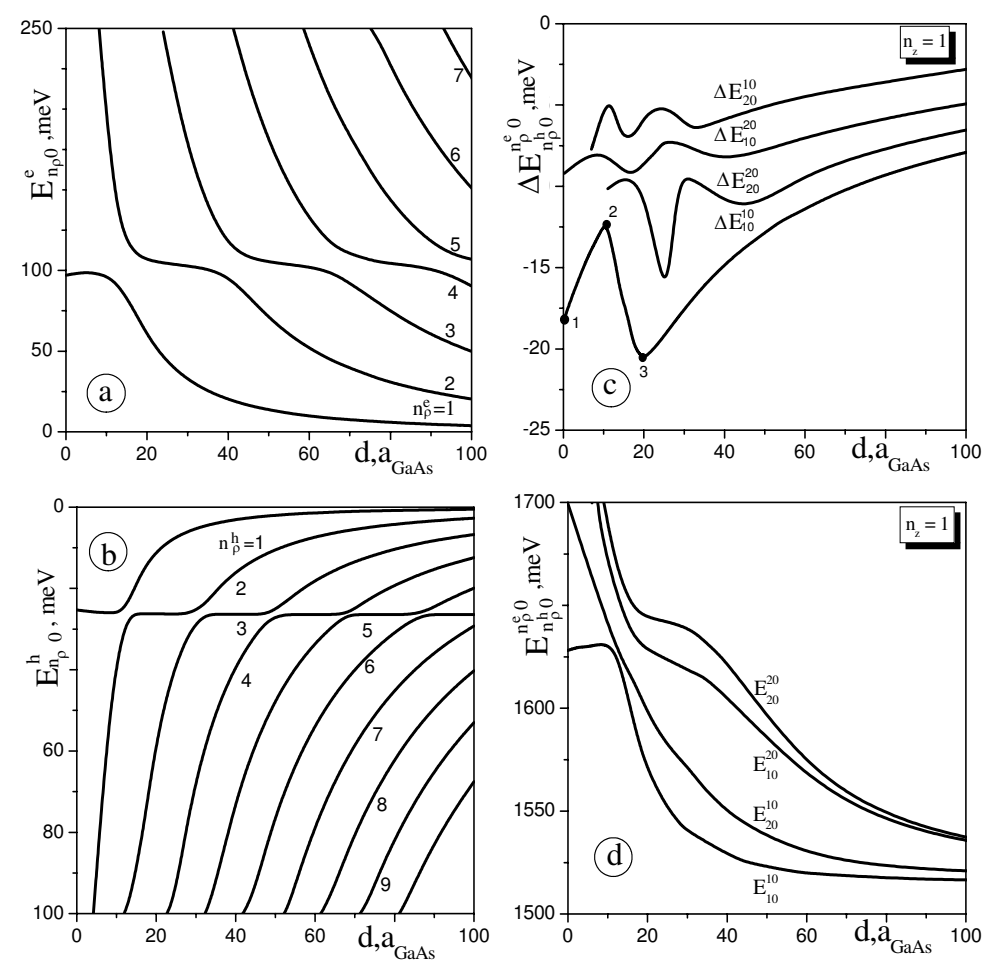

Figure 2. Dependences of electron energy $E_{n_{\rho} 0}^{\mathrm{e}}(\mathrm{a})$, heavy hole energy $E_{n_{\rho} 0}^{\mathrm{h}}(\mathrm{b})$, exciton binding energy $\Delta E_{n_{\rho}^{\mathrm{h}} 0}^{n_{\mathrm{e}}^{\mathrm{e}} 0}$ (c) and exciton energy $E_{n_{\rho}^{\mathrm{h}} 0}^{n_{\rho}^{\mathrm{e}} 0}(\mathrm{~d})$ on the inner wire diameter (d) at $P=0, n_{z}=1, m=0$, and the experimentally obtained barrier-shell width $(\Delta=4 \mathrm{~nm})$ and nanotube width $(h=4 \mathrm{~nm})$ [5]. Points 1, 2, 3 in figure 2 (c) denote the extreme magnitudes of exciton binding energy: point 1 - electron and hole are located in the space of nanotube; point 2 - the hole is located in nanotube and the electron - in nanotube and the inner wire with the probabilities $\approx 0,5$; point 3 - the both electron and hole, with probability close to one, are located in the inner wire.

The new electron and hole energy levels arise when the quantum wire appears and its diameter $d$ increases. The whole spectra shift into the region of lower energies and the anti-crossings of energy levels are observed. The anti-crossing phenomena are caused by the splitting of energy levels due to the tunnel effect present between the quantum wire (with diameter $d$ ) and the nanotube (of $h$ width) through the finite potential barrier (of $\Delta$ width). Both the electron and hole are located in the space of a nanotube at that plots of $E_{n_{\rho} 0}^{(\mathrm{e}, \mathrm{h})}$ dependences on $d$, where the energies of quasi-particles are almost unchanged. The plots where the energies of both quasi-particles rapidly decrease correspond to the states in which the electron and hole are located in the inner wire with the probability close to one. 
The exciton binding energies ( $\Delta E_{n_{\rho}^{\mathrm{h}} 0}^{n_{\mathrm{e}}^{\mathrm{e}} 0}$ ) [figure 2 (c)] non-monotonously depend on the inner wire diameter $d$ taking several minimal and maximal magnitudes for all states. This is clear because when the electron and hole are in their ground states, then, at $d=0$, there is no inner wire, and the both quasi-particles are localized in the space of a nanotube (with $h=4 \mathrm{~nm}$ ) and the binding energy is $\Delta E_{10}^{10} \approx 18 \mathrm{meV}$ [point “1” in figure2 2 (c)].

When the inner wire appears and its diameter increases, the absolute value of the binding energy somewhat decreases because the electron wave function, as the one for the light quasi-particle, increasingly penetrates into the space of the inner wire, while a massive hole does not change its location. Herein, the effective distance between quasi-particles increases. At some critical $d$ [point "2" in figure 22(c)], the massive hole also begins to penetrate into the inner wire. The effective distance between quasi-particles decreases and, consequently, the absolute value of the binding energy increases. The maximal value of the binding energy ( $\Delta E_{10}^{10} \approx 22 \mathrm{meV}$ ) is obtained when both the electron and hole, with probability close to one, are located in the inner wire [point " 3 " in figure 2 (c)]. At a further increase of $d$, the binding energy decreases only, because the effective distance between the electron and hole in the space of the inner wire becomes bigger.
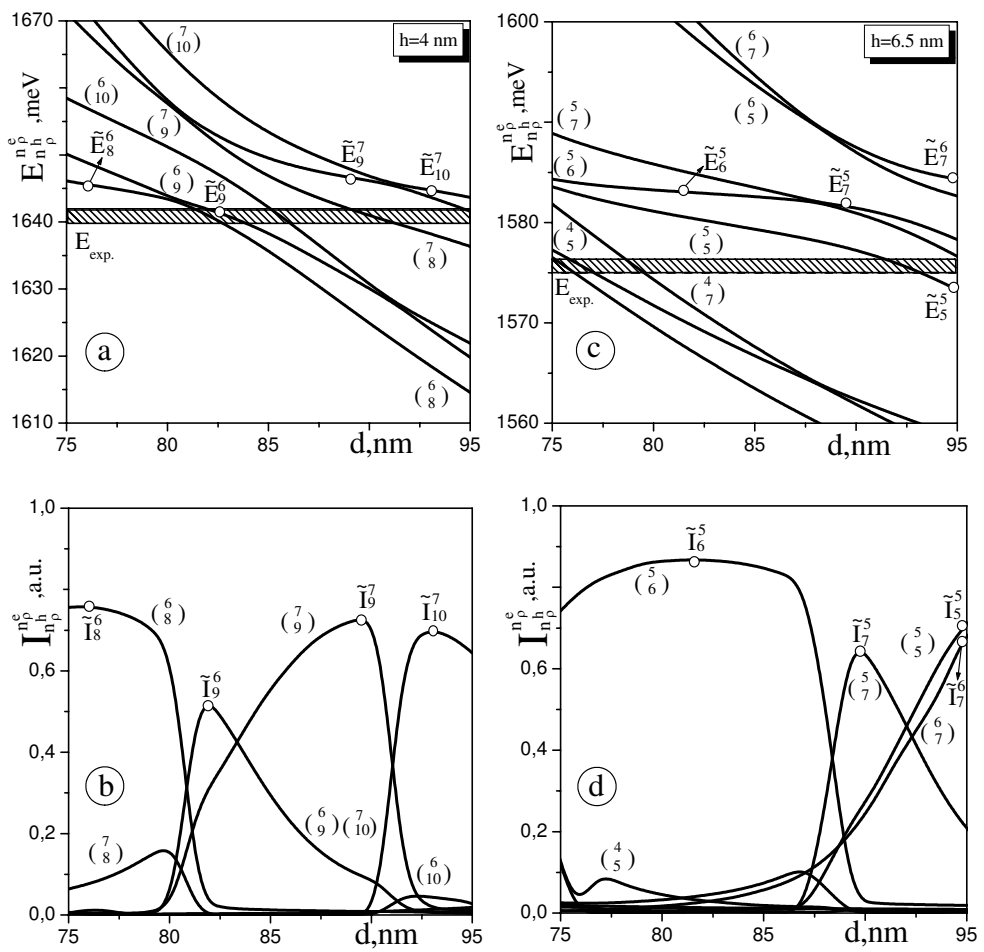

Figure 3. Dependences of exciton energies $E_{n_{\rho}^{\mathrm{h}}}^{n_{\rho}^{\mathrm{e}}}$ and intensities of inter-band quantum transitions $I_{n_{\rho}^{\mathrm{h}}}^{n_{\rho}^{\mathrm{e}}}$ on inner wire diameter (d) in the range: $d=85 \pm 10 \mathrm{~nm}$ at nanotube widths: $h_{1}=4 \mathrm{~nm}(\mathrm{a}, \mathrm{b})$ and $h_{2}=6.5 \mathrm{~nm}$ (c, d). The magnetic quantum number is equal to zero, thus index " 0 " is omitted. The region of energies where the radiation peaks are experimentally observed [8] is shown at the figures by dash strips. The maximal magnitudes of the intensities of quantum transitions and the respective exciton energies are shown by the points.

Similarly, the non-monotonous behaviour of a binding energy of an exciton in its excited states can be explained by the change of an electron and hole location in a quantum wire or nanotube. 
We should note that $V(z)$ potential (16) insomuch well approximates the effective potential (12) that the corrections to the exciton binding energy (26) do not exceed $0.5 \mathrm{meV}$ in all states and for any geometrical sizes of a nanotube.

The exciton energy is two orders bigger than the absolute value of the binding energy. Thus, the dependences of the exciton energies $E_{n_{\rho}^{\mathrm{h}} 0}^{n_{\mathrm{e}}^{\mathrm{e}} 0}$ in the region of low energies on the inner wire diameter $d$, figure 2(d), are completely determined by the peculiarities of electron and hole energies. In particular, the anti-crossing of exciton energies is observed in these functions. This is caused by anti-crossings of electron and hole energy levels.

In order to compare the obtained theoretical results with the experimental ones, in figure 3, the exciton energies $E_{n_{\rho}^{\mathrm{h}}}^{n_{\rho}^{\mathrm{e}}}$ and respective intensities of quantum transitions $I_{n_{\rho}^{\mathrm{h}}}^{n_{\rho}^{\mathrm{e}}}$ are presented as functions of the inner wire diameter $d$ within the ranges: $d=85 \pm 10 \mathrm{~nm}$ and for two nanotube widths: $h_{1}=4 \mathrm{~nm}$ [figure3 (a), (b)] and $h_{2}=6.5 \mathrm{~nm}$ [figure 3](c), (d)]. At these parameters, the luminescence peaks with the energies shown in figures 3 (a), (c) by dashed strips were observed experimentally. The numerical calculations of exciton energies and intensities of quantum transitions are performed at a magnetic quantum number equal to zero, thus index “0” is dropped for convenience.

All figures prove that only some of the exciton curves in the vicinity of experimental data can be characterized by the intensities more than 0.5 . For example at $h_{1}=4 \mathrm{~nm}$ these energies are: $E_{8}^{6}, E_{9}^{6}, E_{9}^{7}$, $E_{10}^{7}$ [figure 3 (a), (b)] and $E_{5}^{5}, E_{6}^{5}, E_{7}^{5}, E_{7}^{6}$ at $h_{2}=6.5 \mathrm{~nm}$ [figure3 (c), (d)]. The maximal intensities $\left(\tilde{I}_{n_{\rho}^{\mathrm{h}}}^{n_{\rho}^{\mathrm{e}}}\right)$ correspond to the exciton energies $\left(\tilde{E}_{n_{\rho}^{\mathrm{h}}}^{n_{\rho}^{\mathrm{e}}}\right)$, at which, evidently, the luminescence peaks should be experimentally observed. Then, depending on the exact experimental sizes of the inner wire, the luminescence peaks observed experimentally [8] can be produced by different exciton states with the energies slightly different from each other at a fixed nanotube width $h$.

Finally, the calculations of probability density of electron and hole location in a nanostructure prove that the both quasi-particles producing the exciton in the above mentioned states $\left(\tilde{E}_{n_{\rho}^{\mathrm{h}}}^{n_{\rho}^{\mathrm{e}}}\right)$ are localized in the nanotube of $h$ width.

\section{Conclusions}

1. The theory of exciton spectra for a multi-shell hexagonal nanotube is developed within the models of effective masses and rectangular potentials for the electron and hole using the method of the effective potential for obtaining the exciton binding energy.

2. The exciton binding energy for all states non-monotonously depends on the inner wire diameter $d$ approaching several minimal and maximal magnitudes. The behaviour of the exciton binding energy is quite well explained by the complicated character of distributions of probability densities of the electron and hole locations in the spaces composition parts of a multi-shell nanotube.

3. The numerical results for the exciton energy spectrum and intensities of quantum transitions, obtained within the developed theory, correlate well with the experimental data for the radiation spectra of $\mathrm{GaAs} / \mathrm{Al}_{0.4} \mathrm{Ga}_{0.6}$ As nanotubes. 


\title{
References
}

1. Suenaga K., Colliex C., Demoncy N., Loiseau A., Pascard H., Willaime F., Science, 1997, 278, 653; doi $10.1126 /$ science.278.5338.653

2. Zhang Y., Suenaga K., Colliex C., Iijima S., Science, 1998, 281, 973; doi 10.1126/science.281.5379.973

3. Persson A.I., Larsson M.W., Stenstro S., Ohlsson B.J., Samuelson L., Wallenberg L.R., Nat. Mater., 2004, 3, 677; doi $10.1038 /$ nmat1220

4. Dubrovskii V.G., Cirlin G.E., Ustinov V.M., Semiconductors, 2009, 43, No 12, 1539; doi 10.1134/S106378260912001X

5. Mohan P., Motohisa J., Fukui T., Appl. Phys. Lett., 2006, 88, 013110; doi 10.1063/1.2161576

6. Mohan P., Motohisa J., Fukui T., Appl. Phys. Lett., 2006, 88, 133105; doi 10.1063/1.2189203

7. Heigoldt M., Arbiol J., Spirkoska D., Rebled J.M., Conesa-Boj C.S., Abstreiter G., Peiro F., Morantece J.R., Fontcuberta i Morral A., J. Mater. Chem., 2009, 19, 840; doi 10.1039/b816585h

8. Fontcuberta i Morral A., Spirkoska D., Arbiol J., Heigoldt M., Morante J.R., Abstreiter G., Small, 2008, 4, 899; doi $10.1002 / \mathrm{smll} .200701091$

9. Kasapoglu E., Sari H., Sokmen I., Surf. Rev. Lett., 2003, 10, 737; doi 10.1142/S0218625X03005566

10. Bouhassoune M., Charrour R., Fliyou M., J. Appl. Phys., 2002, 91, 232; doi 10.1063/1.1419261

11. Sidor Y., Partoens B., Peeters F.M., Phys. Rev. B, 2007, 76, 195320; doi 10.1103/PhysRevB.76.195320

12. Slachmuylders A.F., Partoens B., Magnus W., Physica E, 2008, 40, 2166; doi 10.1016/j.physe.2007.10.091.

13. Tkach M., Makhanets O., Dovganiuk M., Phys. Solid State, 2009, 51, 2529; doi 10.1134/S1063783409120166.

14. Tkach M., Makhanets O., Dovganiuk M., Voitsekhivska O., Physica E, 2009, 41, 1469; doi 10.1016/j.physe.2009.04.018

15. Ogawa T., Takagahara T., Phys. Rev. B, 1991, 44, 8138; doi 10.1103/PhysRevB.44.8138

16. Davies J.H., The Physics of Low-dimensional Semiconductors: An Introduction. Cambridge University Press, New York, 1998.

17. Hai G.Q., Peeters F.M., Devreese J.T., Phys. Rev. B, 1993, 48, 4666; doi 10.1103/PhysRevB.48.4666

\section{Екситонний спектр у багатошаровій шестигранній напівпровідниковій нанотрубці}

\author{
О.М. Маханець, В.І. Гуцул, Н.Р. Цюпак, О.М. Войцехівська \\ Чернівецький національний університет ім.Ю. Федьковича, \\ Україна, 58012 Чернівці, вул.Коцюбинського, 2
}

У наближенні ефективних мас та прямокутних потенціалів, з використанням методу ефективного потенціалу побудовано теорію екситонного спектра у складній багатошаровій шестигранній напівпровідниковій нанотрубці. Отримані теоретичні результати добре пояснюють експериментальні положення піків люмінесценції у нанотрубках GaAs/Al $0.4 \mathrm{Ga}_{0.6} \mathrm{As}$.

Ключові слова: шестигранна нанотрубка, квантовий дріт, екситонний спектр 


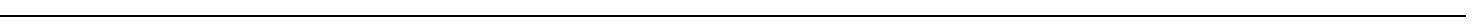

